\title{
Towards Broadening the COVID-19 Knowledge Base
}

When the coronavirus 2019 (COVID-19) pandemic struck during the first quarter of 2020, global attention trained in on the city of Wuhan, the capital of Hubei province in China, where the first cases were reported in December 2019. With its exponential spread to other countries over a short span of time, infecting millions of people and exacting a global death toll of over half a million during the first six months of its discovery, ${ }^{1}$ interest in the little-known yet devastating severe acute respiratory syndrome coronavirus 2 (SARS-CoV-2) virus splurged. As quickly as the virus spread across the globe did the scientific publications on the subject go into circulation, a phenomenon unequalled compared to other pandemics, with an estimated rate of 181 articles published per day. ${ }^{2}$ The scientific community churned as much data as the disease was evolving, covering the range from the epidemiology and clinical symptomatology to treatment and disease prevention. The significant number of deaths occurring among healthcare workers further hit home as the Philippines experienced its share of mortalities, especially of prominent personalities in the medical world. With the scientific literature growing phenomenally, the stage for a global research database on COVID-19 had invariably set in. ${ }^{3}$

The various scientific communities in the Philippines did not also lose time in pursuing research endeavors to bridge the knowledge gaps as these relate to the local setting. Teams from medicine, sociology, mathematics, and other disciplines came together to answer the queries within and across their fields of expertise. ${ }^{4,5}$ The Philippine General Hospital (PGH) as an institution encouraged the pursuit of new knowledge on COVID-19 among its constituents. Support from no less than its Hospital Director, Dr. Gerardo D. Legaspi, came through an allocation of funds in the amount of four million Philippine Pesos solely for COVID-related research.

This Acta Medica Philippina Special Issue is dedicated to the initial researches conducted in the PGH on COVID-19. It chronicles the issues and concerns of the PGH as a COVID Referral Center as it responds to the medical needs of COVID patients while ensuring the safety and protection of its invaluable hospital personnel. The clinical profile and outcomes of patients presenting at the emergency room as well as those admitted to the various units of the hospital such as the intensive care, maternal, and neonatal units are carefully described in four papers. The initial observations on the patient profile as these relate to clinical outcomes are similar to those reported in literature with a poorer prognosis noted among the elderly, those requiring ventilatory support, and those developing complications such as acute kidney injury, stroke, and sepsis. The maternal and neonatal outcomes as documented in this issue have been favorable for the mother-infant dyad with no evidence of vertical transmission of the disease. While children are less likely to be infected by SARS-CoV-2 virus, the local experience in the hospitalized pediatric population during the early phase of the pandemic has highlighted cough and dyspnea as the most common respiratory symptoms. The attempt at determining the correlates for mortality in the included paper was inconclusive, which challenges future researchers to pursue the endeavor in a prospective manner.

In the continual thrust for expanding the methods of diagnosing COVID-19 beyond the standard nasopharyngeal swab reverse transcriptase-polymerase chain reaction (RT-PCR) test, the validation of the snort-spit saliva RT-PCR test in the local setting presents a welcome alternative to the standard manner of specimen collection. With vaccination as an important strategy in the control of COVID-19 infections, up-to-date recommendations on the efficacy and safety of the vaccines are essential in providing guidance and one such living recommendation is available in this issue.

Faced with a highly transmissible disease that can easily wreak havoc to any healthcare system, a review of healthcare delivery processes and the development of more suitable operational guidelines are in order. This issue shares the experience from the UP Health Service and the Department of Obstetrics and Gynecology during the pandemic as they constantly create ways of addressing the new normal. The Department of Surgery on the other hand offers some ethical and operational guidelines to streamline the healthcare delivery system to achieve efficiency. Such operational measures especially for the elective surgical cases could proactively minimize the deleterious outcomes brought about by delays in the management of non-COVID cases, which is underscored in the paper on the treatment of patients with osteosarcoma. Operational guidelines for aerosolgenerating procedures such as gastrointestinal endoscopy have been advanced in many centers abroad and one paper in this issue integrates the current local and international guidelines for use in resource-limited endoscopy units in the country. 
Amid the challenges that the COVID-19 pandemic has wrought on the healthcare system, opportunities to creatively manage the health concerns of the patients have surfaced, one of which is telemedicine. Telemedicine has long been utilized in the country before the pandemic in the geographically isolated and disadvantaged areas where there is a dearth of practicing specialists. Using the Community Health Information Tracking System (CHITS) and the RxBox Telehealth Device developed by the University of the Philippines (UP) National Telehealth Center in collaboration with the UP Diliman College of Engineering, selected rural health centers in the Philippines have been accorded the support for assisted individualized case management. ${ }^{6}$ With the physical restrictions for face-to-face consultations, compounded further by the protracted nature of the pandemic, telemedicine may yet be an essential strategy in bridging the gaps in the current healthcare delivery system. Described in this Special COVID issue are two articles on the relevance of telemedicine in the local setting, one that appertains to the general health services among healthcare personnel in the hospital setting and the other on the care of cancer patients served by the hospital. New process flows need to be meticulously mapped out to ensure service efficiency and patient satisfaction. As the COVID-19 crisis lays open numerous uncertainties to the world at large, one cannot but take confidence on what Albert Einstein once said, "In the midst of every crisis, lies great opportunity."

\author{
Juliet Sio Aguilar, MD, MSc \\ Former Professor and Past Chair \\ Department of Pediatrics \\ Philippine General Hospital \\ University of the Philippines Manila
}

\title{
REFERENCES
}

1. Pitlik SD. COVID-19 compared to other pandemic diseases. Rambam Maimonides Med J. 2020 Jul; 11(3):e0027.

2. Lui A. Letter to the Editor: Exponential increase in COVID-19 related publications compared to other pandemic diseases. Rambam Maimonides Med J. 2021 Jan; 12(1):e0009.

3. World Health Organization [Internet]. Global research on coronavirus disease (COVID-19). [cited 2021 Apr]. Available from: https://www.who. int/emergencies/diseases/novel-coronavirus-2019/global-research-on-novel-coronavirus-2019-ncov

4. Aguilar FV. Preparedness, Agility, and the Philippine Response to the Covid-19 Pandemic: The Early Phase in Comparative Southeast Asian Perspective. Philippine Studies: Historical and Ethnographic Viewpoints. 2020; 68 (3-4): 373-421.

5. David G, Rye RS, Agbulos MP. COVID-19 Forecasts in the Philippines: Insights for Policy Making [Internet]. [cited 2021 Apr]. Available from: https://up.edu.ph/covid-19-forecasts-in-the-philippines-insights-for-policy-making-updated-as-of-april-22-2020/

6. Department of Science and Technology Region X [Internet]. Roll-out of 1000 RxBox Telehealth Device in Selected Rural Health Centers in the Philippines. [cited 2021 Apr]. Available from: https://region10.dost.gov.ph/101-roll-out-of-1000-rxbox-telehealth-device-in-selected-rural-healthcenters-in-the-philippines 